\title{
Analysis of Biased PET Images Caused by Inaccurate Attenuation Coefficients
}

\author{
Young-Don Son ${ }^{1}$, Hang-Keun Kim ${ }^{1}$, Sung-Tae Kim ${ }^{1}$, Nam-Beom Kim ${ }^{1}$, Young-Bo Kim ${ }^{1}$, and Zang-Hee Cho ${ }^{1,2}$ \\ ${ }^{I}$ Neuroscience Research Institute, Gachon University of Medicine and Science, Incheon, South Korea; and ${ }^{2}$ Department of \\ Radiological Sciences, University of California, Irvine, California
}

PET scanners with an elongated axial field of view intended to increase overall system sensitivity, such as the high-resolution research tomograph (HRRT) scanner, have been reported to produce images with decreased signals in the brain stem and cerebellum. The cause of this negative bias of the images was analyzed, and the effects of an inaccurate linear attenuation coefficient ( $\mu$-value) of tissue and bones were separately examined. Methods: A new phantom was manufactured, and 18 human subjects were recruited for the study. ${ }^{18} \mathrm{~F}-\mathrm{FDG}$ PET images were reconstructed using attenuation coefficient maps generated by various algorithms. The algorithms included maximum a posteriori reconstruction for transmission data (MAP-TR) with default priors, MAP-TR with adjusted priors for bone (MAP$\mathrm{TR}_{\mathrm{adj}-\mathrm{b}}$ ), MAP-TR with adjusted priors for tissue (MAP-TR $\mathrm{Rdj}_{\mathrm{t}}$ ), and noise-equivalent count TR and CT-TR. Results: With the

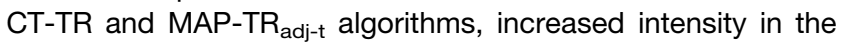
brain stem and cerebellum was seen, and negative bias was reduced. With the MAP-TR adj-t $_{\text {algorithm, however, positive bias }}$ increased in the central region. Inappropriate attenuation coefficients of brain tissue increased the positive or negative bias of reconstructed images, especially for the central regions of the volume. Poor representation of the skull or bone also locally increased the bias in the near regions where bone detection had failed. Conclusion: An inaccurate $\mu$-map obtained from the MAP-TR algorithm caused the bias problem for the HRRT system. The CT-TR algorithm provided a relatively more reliable $\mu$-map that demonstrated a small degree of intensity bias. Appropriate priors for $\mu$-values of each tissue compartment and better classification to distinguish bone from tissue are necessary for accurate attenuation correction.

Key Words: PET/CT; HRRT; three-dimensional PET; attenuation correction; scatter correction

J Nucl Med 2010; 51:753-760

DOI: 10.2967/jnumed.109.070326

\section{S} the emergence of PET $(1,2)$ in medical imaging, great advances in the performance of the modality have been made continuously to observe the nature of brain activity in vivo with sufficient resolution and sensitivity.

Received Sep. 5, 2009; revision accepted Feb. 12, 2010.

For correspondence or reprints contact: Zang-Hee Cho, Neuroscience Research Institute, Gachon University of Medicine and Science, 1198

Kuwol-Dong, Namdong-Gu, Incheon, 405-760, South Korea.

E-mail: zcho@gachon.ac.kr

COPYRIGHT @ 2010 by the Society of Nuclear Medicine, Inc.
The development of new detector materials with better characteristics, such as stopping power and light output, has resulted in the fundamental improvement of PET systems. Bismuth germanate and lutetium oxyorthosilicate (3) are representative detector materials that are currently used. A decreased detector width and gantry diameter have enhanced the spatial resolution and the sensitivity, respectively, of the PET systems. Furthermore, a 3-dimensional (3D) PET scanner, which collects additional oblique lines of response by removing septa between the rings, has been proposed and implemented to increase system sensitivity (4). The most advanced commercial 3D PET scanner is the high-resolution research tomograph (HRRT; CTI/Siemens) dedicated to brain research. This state-of-the-art PET scanner can achieve spatial resolution as high as $2.5 \mathrm{~mm}$ (5), as expected by the use of small detectors. A small ring diameter and a long axial field of view (FOV) maximize the sensitivity for brain imaging.

Despite the advancements, the HRRT still has some limitations, including increased scatter, which is the typical problem with the $3 \mathrm{D}$ scanners (6). In addition, relatively lower signal intensity in the cerebellum and brain stem was recently reported by some HRRT users. This lower signal intensity has been described in the Alzheimer's Disease Neuroimaging Initiative PET Core documents by Robert Koeppe in which the pons was reported to show an intensity approximately $12 \%$ lower than the global mean (http:// www.loni.ucla.edu/twiki/bin/view/ADNI/ADNIPETCore). Koeppe has postulated that the possible cause of this problem is related to scatter correction or attenuation correction incorporated with the HRRT.

Recently developed PET/CT scanners can provide better $\mu$-maps in terms of spatial resolution and signal-to-noise ratio among commercial PET systems by using CT images. $\mu$-values have nonlinear characteristics when converting the $\mu$-values measured with the low energy of $x$-rays to the $511 \mathrm{keV}$ of PET. The relationship of these energy-dependent $\mu$-values was approximated to the bilinear model, and this approximation has been successfully applied for the attenuation correction of PET/CT. Radionuclide-based transmission data, such as with ${ }^{68} \mathrm{Ge}$ or ${ }^{137} \mathrm{Cs}$, have less or no energy-dependent limitation, but the $\mu$-map quality is much poorer. This poor $\mu$-map quality becomes worse as 
the scanning time of the transmission data becomes shorter. With a decreased detector size for higher resolution such as with the HRRT, the counting rate of each detector severely drops. To overcome a poor counting rate or signal-to-noise ratio of a $\mu$-map for the HRRT, the maximum a posteriori reconstruction algorithm for transmission data (MAP-TR) has been adopted. MAP-TR is a Bayesian reconstruction algorithm for transmission data with predefined smoothness and intensity priors, such as for air, cavity, tissue, and bone (7). However, van Velden et al. (8) reported that default priors in the HRRT system resulted in a thicker skull on the $\mu$-map, and this situation might result in an incorrect attenuation correction. van Velden et al. (8) recommended MAP-TR with newly adjusted priors of bone (MAP-TR adj-b $_{\text {) }}$ and noise-equivalent count (NEC)-TR with segmentation as the transmission reconstruction algorithms for the HRRT. NEC-TR applies NEC scaling to the measured blank over transmission sinogram data to restore pseudoPoisson distribution (9). With these algorithms, the skull on the $\mu$-map became thinner, but the 2 algorithms could not solve the intensity drop in the lower region and the underlying cause of the problem was not clearly identified. More recently, an HRRT user group developed a new transmission reconstruction algorithm that considered the scattering effect of the transmission data. The algorithm, however, will not be evaluated in this article because it has not been published yet.

The $\mu$-values used for the brain in many PET studies have, interestingly, varied in a range from 0.095 to 0.099 $\mathrm{cm}^{-1}(10,11)$. The theoretic $\mu$-value for brain tissue has been reported as $0.0994 \mathrm{~cm}^{-1}$ at $511 \mathrm{keV}$ according to an International Commission on Radiation Units and Measurements report (12). However, the HRRT uses 0.096 $\mathrm{cm}^{-1}$ as a default prior for brain tissue.

In the current study, the effects of defective $\mu$-maps on image intensity were analyzed separately for bone and brain tissue components. Transmission reconstruction methods originally incorporated with the HRRT system and modified priors were evaluated and were quantitatively compared for a newly designed phantom and for human subjects. A PET/CT system was used to provide reference images to compare the intensity of the negative bias. A long transmission scan with positron emission sources provides a better reference image than does a CT transmission scan, but long radioactive exposure for human subjects limits the scanning. We, therefore, assumed that despite the possible conversion errors between CT Hounsfield units to $511-\mathrm{keV}$ $\mu$-values depending on the materials, the expected error is negligible in human scanning.

\section{MATERIALS AND METHODS}

HRRT PET and PET/CT systems were used for the study. Detailed descriptions of the 2 systems are given in Supplemental Table 1 (supplemental materials are available online only at http:// jnm.snmjournals.org).

\section{Phantom and Subjects}

Neuroscience Research Institute (NRI) Attenuation Phantom. To evaluate the attenuation-correction methods quantitatively, we designed and manufactured a new attenuation phantom. The basic design of the phantom was motivated by the $\mu$-map of the human skull, of which some structures in the lower part are not represented on the $\mu$-map. Teflon (DuPont) was chosen as the material for the outer shell, to represent an artificial skull. Although it has been reported that the bilinear method used for PET/CT does not work appropriately for Teflon (13), Teflon has the closest $\mu$-value to the skull among the available materials at the energy level for both x-rays and $\gamma$-rays. The outer shell has 2 different thicknesses. For the hemispheric part, the skull was made thicker and the base was made thinner; for the cylindric part, the front was made thinner and the back was made thicker (Supplemental Fig. 1). This design reflects the detectable and undetectable parts among the bones for the transmission data.

Human Subjects. Data for 18 subjects were used for this study. All subjects were men who did not have a neurologic disease. Nine subjects were scanned with the PET/CT system, and 9 subjects were scanned with the HRRT. For the subjects scanned with HRRT, CT images were shortly acquired for the transmission data using PET/CT. All procedures were approved by the Internal Review Board of Gachon University of Medicine and Science.

\section{Transmission Data Acquisition and Reconstruction}

Transmission scanning was performed using a typical human brain-scanning protocol (Supplemental Table 2).

The representative 4 transmission reconstruction algorithms implemented in the HRRT system and the bilinear transformation CT method were compared (NEC-TR with segmentation, MAPTR with default priors $[$ MAP-TR def $]$, MAP-TR $_{\text {adj-b }}$, and MAP-TR with adjusted tissue priors $\left[\mathrm{MAP}-\mathrm{TR}_{\mathrm{adj}-\mathrm{t}}\right]$ and CT-based $\mu$-map [CT-TR]).

The reconstruction parameters for the radionuclide-based transmission are described in Supplemental Table 3. HRRT with the MAP-TR algorithm used the scaling methods to adjust the biased attenuation coefficients due to the different energy of ${ }^{137} \mathrm{Cs}$ at 662 $\mathrm{keV}$ (supplemental materials). CT-TR was prepared by homemade software converting the Hounsfield units of CT to the $\mu$-value at $511 \mathrm{keV}$. A single CT energy-scaling method based on bilinear transformation (14) was used. The converted CT-based $\mu$-map was coregistered onto the ${ }^{137} \mathrm{Cs}$-based $\mu$-map reconstructed by the MAP-TR algorithm using the Vinci software (Max-Planck Institute). Six-parameter rigid-body affine transformation was used for the coregistration, and mutual information was selected as the similarity measurement. To match the resolution of the CT-based $\mu$-map with the HRRT image, smoothing with a 3D gaussian filter with a $3-\mathrm{mm}$ full width at half maximum was performed.

\section{Emission Data Acquisition and Reconstruction}

${ }^{18}$ F-FDG PET was performed for the human subjects and phantom using the same protocol in both PET scanners (supplemental materials).

The 3D ordinary Poisson ordered-subset expectation maximization (OP-OSEM3D) algorithm accelerated by the parallelized computations (15) was used for the reconstruction of the HRRT images. The parameters of the OP-OSEM3D algorithm were as follows: $256 \times 256 \times 207$ image voxels, 6 iterations, and 16 subsets. The PET/CT scanner used the 2-dimensional OSEM algorithm, and the parameters were as follows: $256 \times 256 \times 81$ 
image voxels, 8 iterations, and 16 subsets. Normalization, random correction, attenuation correction, and scatter correction were applied with the reconstruction method in both scanners. A $\mu$-map for attenuation correction was also used for estimating the scatter effects. Scatter-correction factors were calculated using the single-scatter simulation method (10) with a $\mu$-map for both scanners.

After reconstruction, all reconstructed images were spatially normalized to the Montreal Neurological Institute template and were smoothed with a gaussian kernel of $8 \mathrm{~mm}$ in full width at half maximum using SPM2 (Wellcome Trust Centre for Neuroimaging).

\section{Data Analysis}

Comparison of Intensity Uniformity in Phantom. To compare the uniformity of each reconstruction algorithm, a 2-dimensional region of interest was chosen in each plane using the threshold method. Average pixel intensity of the region of interest was evaluated and plotted along the planes. The intensity ratio of the lower plane over the upper planes was calculated to quantify the uniformity, that is:

$$
\text { Intensity uniformity }(\%)=\frac{\left(\mathrm{I}_{\text {Upper }}-\mathrm{I}_{\text {Lower }}\right)}{\mathrm{I}_{\mathrm{Upper}}} \times 100 \text {. }
$$

The phantom was placed at the middle of the axial field of view ( $\sim 50$ th to $\sim 145$ th planes). $\mathrm{I}_{\text {Upper }}$ and $\mathrm{I}_{\text {Lower }}$ were the averages of the upper planes $(\sim 62$ th to $\sim 92$ th planes) and the lower planes $(\sim 112$ th to $\sim 142$ th planes), respectively.

Statistical Comparison of PET/CT Versus HRRT in Human Subjects. Unlike the phantom study, we used a statistical comparison for human subjects because it was difficult to define the standardized uptake value for each region. A statistical comparison was used to investigate the negative bias of the HRRT images reconstructed by $\mu$-maps for each algorithm. PET/CT was chosen as the reference system, because it is known to be a quantitatively accurate system and has been clinically proven. In particular, PET/ CT has not been reported to have a regional intensity bias. The 2-sample $t$ test was performed using SPM2. Global mean scaling was used to normalize the mean of the intensity between PET/CT and HRRT images.

Intensity Recovery Ratio (IRR). Reconstructed HRRT images were compared to investigate the effect according to $\mu$-maps obtained by each transmission reconstruction method. The IRR was calculated using the following equation:

$$
\operatorname{IRR}(\%)=\frac{\mathrm{I}_{\mathrm{New} \mu-\mathrm{map}}-\mathrm{I}_{\mathrm{MAP}-\mathrm{TR}_{\text {def }}}}{\mathrm{I}_{\mathrm{MAP}-\mathrm{TR}_{\text {def }}}} \times 100,
$$

where $\mathrm{I}_{\text {MAP-TR }}$ and $\mathrm{I}_{\mathrm{New}} \mu$-map are the reconstructed images from the MAP-TR def $_{\text {and }}$ and the new $\mu$-map, respectively.

Analysis of Bone Effect and Tissue Effect. To analyze how the missing or misestimated structures on the $\mu$-maps change the intensity of the HRRT images, 2 simple simulations were used.

Bone effect was simulated for the human data. The $\mu$-maps for the MAP-TR and CT-TR algorithms were segmented as the following 2 compartments: bone and nonbone tissue. This segmentation was accomplished using a simple constant-threshold method. A constant $\mu$-value of $0.105 \mathrm{~cm}^{-1}$ was used as the threshold, and

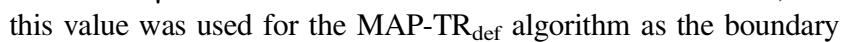
between bone and nonbone tissue. Nonbone tissue were replaced by air $\left(0.0 \mathrm{~cm}^{-1}\right)$ to calculate only the contribution of bone for the attenuation correction. The resultant bone $\mu$-map for the MAP-TR and CT-TR algorithms was converted to attenuation-correction factors (ACFs) as a sinogram form. To roughly visualize the brain location affected by the difference in these 2 bone $\mu$-maps (the socalled bone-effect), the MAP-TR ACFs were subtracted from CTTR ACFs and then backprojection was performed. One sample $t$ test was performed on the subtraction images.

Tissue effect was simulated for the NRI phantom data to see how wrong $\mu$-values of tissue affect the intensity of reconstructed images. The CT-TR $\mu$-map for the phantom was segmented as water and Teflon using the threshold method. The $\mu$-value of the water region for the CT-TR $\mu$-map was replaced by a given value for a range from 0.066 to $0.111 \mathrm{~cm}^{-1}$. With the modified CT-TR $\mu$-map, each image was reconstructed using the same protocol.

\section{RESULTS}

\section{Comparison of $\mu$-Maps According to Transmission Reconstruction}

For human subjects, a CT-based $\mu$-map can describe the fine structure of the skull with high resolution. All algorithms based on the use of ${ }^{137} \mathrm{Cs}$ transmission scan data failed to detect thin or low-density bone structures, which exist mainly in the lower part of the skull (Fig. 1A). The MAP-TR $_{\text {def }}$ algorithm resulted in a $\mu$-map with a thicker and more smeared skull than that with the other algorithms. The MAP-TR ${ }_{\text {adj-b }}$ algorithm generated a $\mu$-map with adequate thickness in the skull, but many bones below the skull except for the teeth had disappeared on $\mu$-images. The NEC-TR algorithm produced a $\mu$-map similar to the one produced by the MAP-TR adj-b $_{\text {algorithm but with }}$ a flattened and thicker skull as seen on $\mu$-images. The $\mu$-values of bone were the highest in the $\mu$-images of the CT-TR and MAP-TR ${ }_{\text {adj-b }}$ algorithms (Fig. 1B). In the case of tissue, the $\mu$-values for the CT-TR and MAP-TR adj-t $_{\text {t }}$ algorithms were approximately $0.0994 \mathrm{~cm}^{-1}$, and the $\mu$-values for the other algorithms were $0.096 \mathrm{~cm}^{-1}$ (Fig. 1C).

For phantom data, ${ }^{137} \mathrm{Cs}$-based $\mu$-maps showed a thicker structure for the 5-mm shell than did the CT-based $\mu$-map, and the 2-mm shell was almost not detected on the $\mu$-map, as was similar for the human subjects (Fig. 1A). Even the 5-mm shell was partially missed or disappeared with

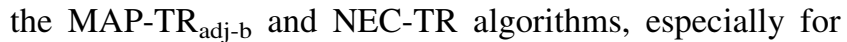
the lower part of the phantom that corresponded to the brain stem. These areas were more smeared on $\mu$-images with the

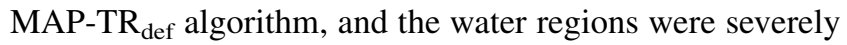
contaminated by the higher $\mu$-values scattered from the Teflon. Measured $\mu$-values of Teflon were the highest on the CT-based $\mu$-maps (Fig. 1B). In contrast, for water the $\mu$-values were about same as the known value, $0.096 \mathrm{~cm}^{-1}$, using all algorithms including CT-TR.

\section{Comparison of Intensity Uniformity in Reconstructed Emission Images}

For the water-filled phantom, each plane of the reconstructed image should ideally have the same intensity. As 


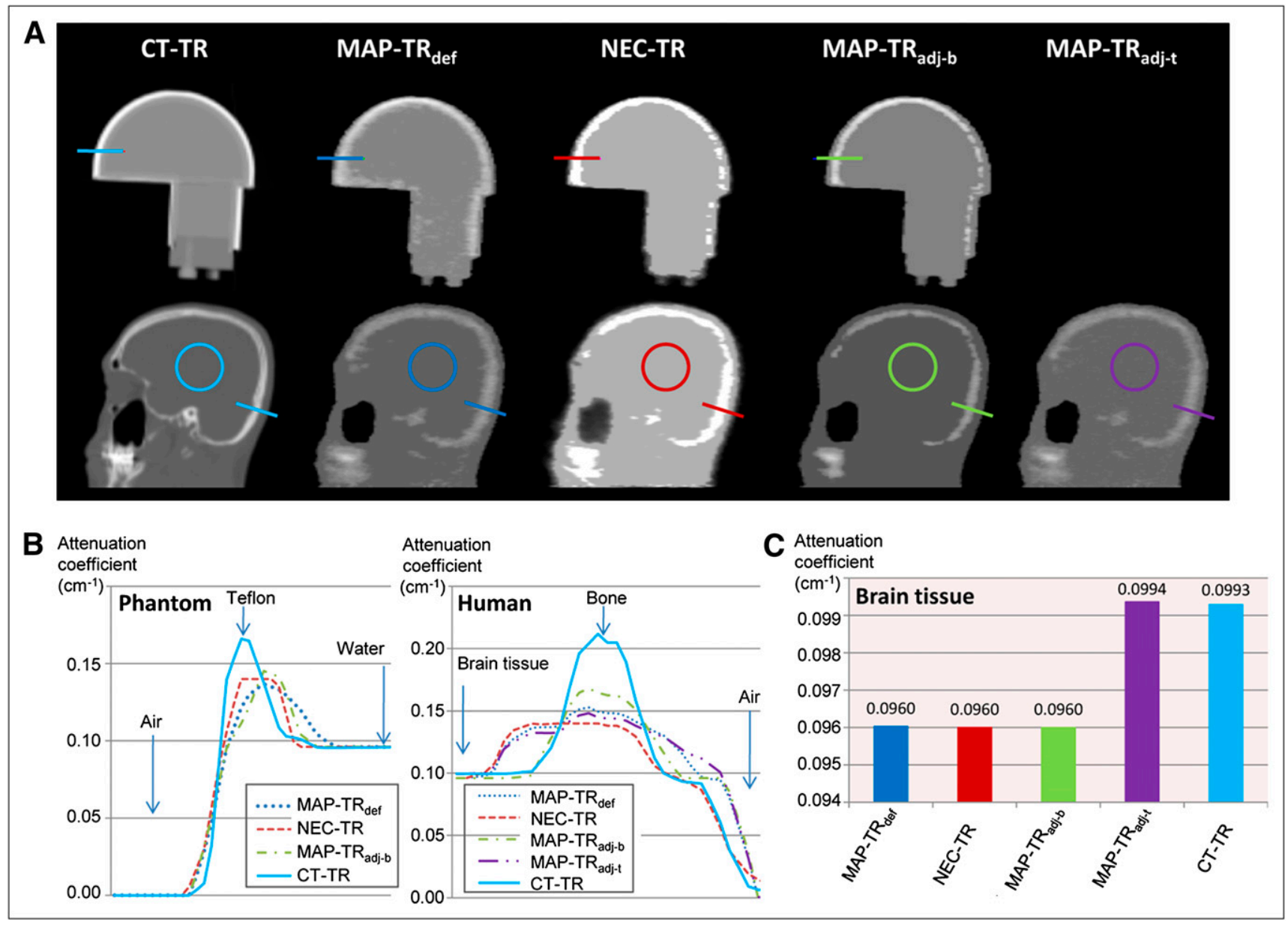

FIGURE 1. (A) Sagittal views of $\mu$-maps according to transmission reconstructions. Upper $\mu$-maps are for NRI phantom and lower ones for human. (B) $\mu$-value profiles of colored lines of $A$. (C) Comparison among mean $\mu$-values of brain tissue. Pixels in colored circles of $A$ are used to obtain mean $\mu$-values for C. CT-TR algorithm provides most accurate $\mu$-values for brain tissue and shape for bone.

shown in Figure 2, a reconstructed image using the CT-TR algorithm showed less than a $2 \%$ difference in intensity between the upper and lower planes along the axial direction. The use of the other algorithms resulted in a more than $5 \%$ decrease in signal intensity at the lower slices. That finding was in accord with the fact that only the CTTR algorithm can represent the thinner shell of the phantom on the $\mu$-map. Although the CT-TR algorithm generates the most accurate $\mu$-map, the small discrepancy between the upper and lower slices was caused by the measured $\mu$-value of water. Interestingly, the values were apparently different between the upper and lower parts of the phantom, at a mean of 0.0961 and $0.0954 \mathrm{~cm}^{-1}$, respectively. This difference was also shown for $\mathrm{HU}$ in the original CT image. In addition, transformation of the $\mu$-values for PET from lower-energy CT data is not accurate with a material such as Teflon.

Bias of the reconstructed HRRT PET images, as compared with PET/CT images for human subjects, is shown in Figure 3. Most of the lower regions of the brain showed negative $t$ values, and the upper regions showed positive $t$ values, regardless of the transmission algorithm. The MAP$\mathrm{TR}_{\mathrm{def}}$ algorithm showed the most significantly negative $t$ values in the lower brain. Overall, the CT-TR algorithm showed the least significant $t$ values for both positive and negative $t$ values, which provided images most similar to the PET/CT images. With the MAP-TR adj-t $_{\text {algorithm, as }}$ compared with the other algorithms, the negative $t$ values were significantly reduced. In contrast, however, positive $t$ values were more increased using the MAP-TR $\mathrm{def}_{\text {algo- }}$ rithm for the middle part of the brain.

To evaluate the appearance of intensity changes with each algorithm, as compared with the MAP-TR def algorithm, the IRR of all subjects was averaged.

Global intensity scaling of a PET image can roughly normalize the intensity of a PET image according to the use of algorithms. The paired $t$ test was used to evaluate the relative change of intensity for each subject with global intensity scaling as shown in Figure 4. The NEC-TR and MAP-TR $_{\text {adj-b }}$ algorithms showed a slight increase in the 


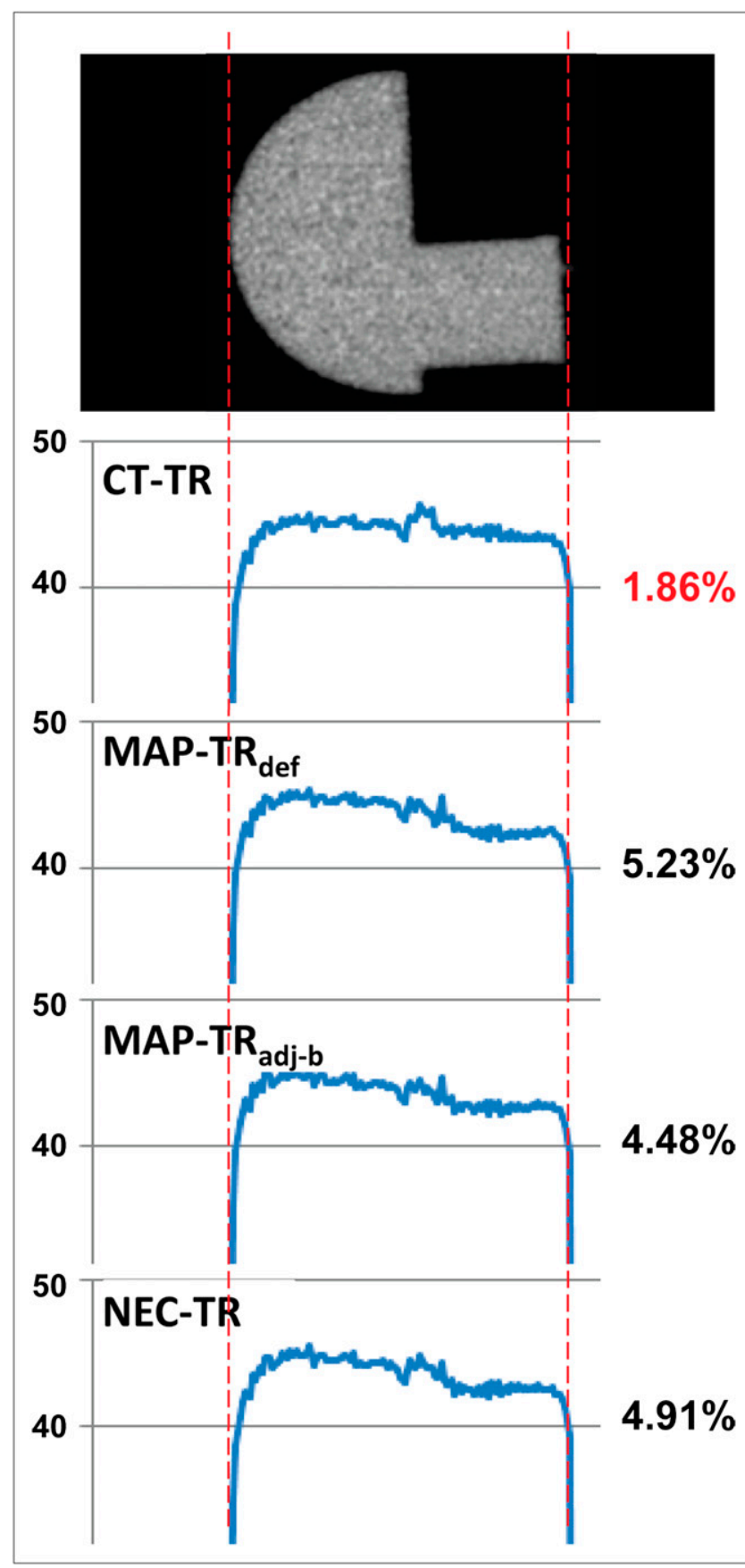

FIGURE 2. Intensity ratio of upper and lower part of phantom in reconstructed PET image according to transmission reconstruction. CT-TR showed most uniform intensity ratio in phantom.

medial regions of the brain and a slight increase in the brain stem and the cerebellum. The MAP-TR adj-t $_{\text {and CT-TR }}$ algorithms showed a more significant and localized increase. The MAP-TR adj-t $_{\text {algorithm resulted in increased }}$ intensity in the central regions of the brain including the brain stem and decreased intensity in the neocortex of the brain. The CT-TR algorithm also resulted in increased intensity in the brain stem and the cerebellum but not in the central regions of the brain. The compensation pattern for

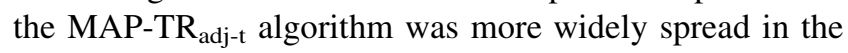
central region of the brain, and the compensation pattern for the CT-TR algorithm was more localized in the brain stem and the cerebellum.

\section{Simulation of Bone Effect and Tissue Effect}

The CT-TR algorithm could represent more bone structures in the lower regions not represented with the MAP$\mathrm{TR}_{\text {def }}$ algorithm (Fig. 1). As compared with the MAP-TR ${ }_{\text {def }}$ algorithm, the skull as seen with the CT-TR algorithm was thinner, but the $\mu$-values were higher. The influence on the reconstructed image of these differences in the bone parts of the $\mu$-maps between CT-TR and MAP-TR def $_{\text {algorithms }}$ was clearly shown in Figure 5. The positively higher $t$ value demonstrates that the CT-TR algorithm produced a consistently higher ACF in most subjects than did the MAP-TR def $_{\text {f }}$ algorithm and vice versa for a negatively higher $t$ value. A positively higher $t$ value was observed in the brain stem and the cerebellum, whereas a negative $t$ value was observed in the middle of the brain. These $t$ values meant that region of the brain stem and the cerebellum had the higher intensity in the reconstructed image by attenuation correction using the CT-TR algorithm than using the MAP-TR def algorithm. This analysis represents only bone effect.

Unlike bone effect, tissue effect in the CT-TR algorithm can be measured more quantitatively using the phantom as shown in Figure 6. The CT-TR $\mu$-map is used as a template for simulating the effect of wrong $\mu$-value of the tissue. The ratio between the upper and lower regions was linearly proportional to the priors. The upper slices and the lower slices were identical when the $\mu$-value of water became $0.090 \mathrm{~cm}^{-1}$ for the simulation results. Discrepancy in this value might be due to the effect of the inaccurate $\mu$-value of Teflon using the CT-TR algorithm, as previously described.

Although the value was much lower than the theoretic $\mu$-value of water $\left(0.096 \mathrm{~cm}^{-1}\right)$, this simulation confirmed that the wrong priors for tissue could influence the ratio of the upper and lower slices. This finding indicates that deeper regions of the brain are more susceptible to an incorrect $\mu$-value of brain tissue.

\section{DISCUSSION}

Attenuation and scatter correction are the most important correction processes of PET for quantitative and diagnostic analysis. These corrections, however, can be biased because of the incorrect estimation of the attenuator. Attenuation correction is more susceptible to the misestimation of attenuation than is scatter correction, because attenuation correction corrects by multiplication whereas scatter correction corrects by subtraction. Inaccurate $\mu$-values can be generated during acquisition or reconstruction of transmission data. Because of the poor counting statistics of the radionuclide source-based transmission scan, thin bones-especially bones in the lower part of the skull-are often misclassified as tissue on 
FIGURE 3. Positive and negative bias of HRRT image, compared with PET/CT image, according to transmission reconstruction. Most significant negative bias was found in lower parts including brain stem and cerebellum as reported (blue circle). Middle parts, such as superior regions of cerebellum and deep occipital cortex, showed significant positive bias (red circle). Both positive and negative biases were minimal in CT-TR. MAP-TR adj-t $_{\text {reduced }}$ negative bias effectively; in contrast, positive bias increased most.

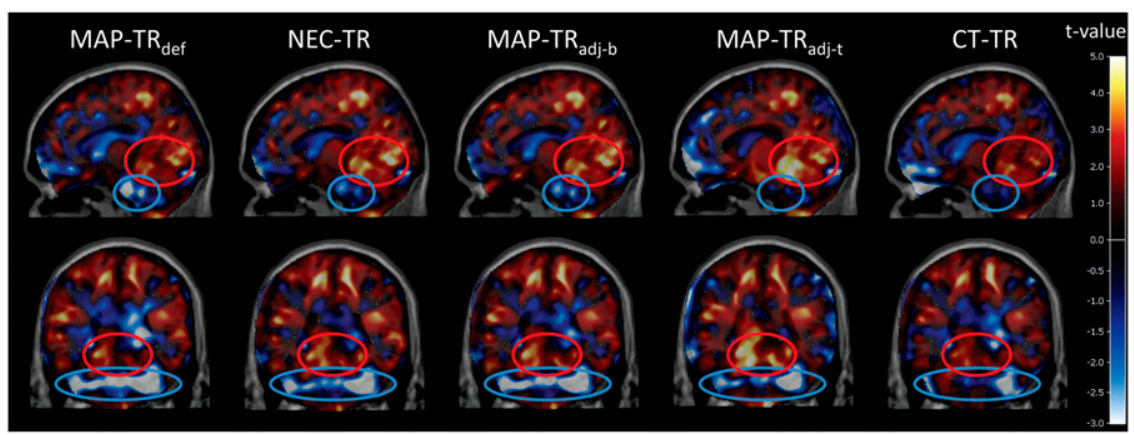

$\mu$-maps. In addition, because of the defects of the transmission reconstruction algorithms, $\mu$-maps can be incorrectly estimated. For the MAP-TR algorithm, for instance, wrong prior information could lead to inaccurate assignment of $\mu$-values. Our studies were basically designed on the basis of these 2 postulations. To simulate these postulations, we designed a new phantom with different volumes in the upper and lower parts of the skull to evaluate uniformity.

As expected, reconstructed PET images using $\mu$-maps generated with the CT-TR algorithm were the most uniform for both phantom and human data. This finding can be explained because of tissue effect and bone effect.

The influence of the misestimated $\mu$-value of tissue on PET images was shown for the simulation with the phantom study. As the defined prior of water was forcibly set to a higher or lower $\mu$-value, signal increase or decrease, respectively, of a PET image for the upper part of the skull became larger than for the lower part of the skull (Fig. 6A). This finding might be due to a larger volume in the upper part of the skull. When ACFs were calculated for each sinogram bin, an erroneous $\mu$-value accumulated along an integral path. Thus, the upper part with a larger diameter was more influenced by the misestimated $\mu$-value. If a spheric phantom was used, the most influenced part became the center of the phantom.
Adjustment of the intensity priors of brain tissue to the ideal values $\left(0.0994 \mathrm{~cm}^{-1}\right)$ increased the signal for the entire area, as shown in Figure 4C. As compared with the MAP-TR def $_{\text {algorithm, the intensity of central regions }}$ including the limbic regions and brain stem showed the greatest increase. The relative signal change in the brain was decreased in the outer cortices and was increased in the central regions. This change restored the intensity in the negatively biased regions with the MAP-TR def $_{\text {algorithm, }}$ but some areas near central regions of the brain, which should not be increased, were contrarily increased too much (Fig. 3D). With the CT-TR algorithm, as compared with the MAP-TR adj-t algorithm, the intensity bias was stabilized in a more balanced way (Fig. 3E). The CT-TR algorithm has almost the same $\mu$-values as the MAP-TR adj-t algorithm for brain tissue, but the restored regions vary slightly. Negative bias in the brain stem and cerebellum was restored, and an excessive increase in the central regions was not shown. It is clearly shown in Figure 4D that increased regions are localized only in the brain stem and cerebellum and not in the central regions. These findings imply that the CT-TR algorithm should have a factor other than tissue effect. Accurate and detailed representation of bone structures, especially for the lower part of skull, is seen with the CT-TR algorithm, as compared with the other MAP-TR algorithms. Effects of missed bones on the $\mu$-map for image intensity as shown in Figure 5 may explain why

FIGURE 4. Change of averaged IRR, compared with MAP-TR def $_{\text {algorithm, }}$ after normalization of average intensity of whole brain for each subject. NEC-TR and MAP-TR adj-b $_{\text {increased intensity of }}$ PET image in most regions of brain, although amount of recovery ratio was not significant. Intensity of central brain regions was increased in MAP-TR adj-t, $_{\text {, }}$ and intensity of outer cortices was decreased. Significantly increased regions of CT-TR were localized in the brain stem and cerebellum and the central regions were slightly changed.

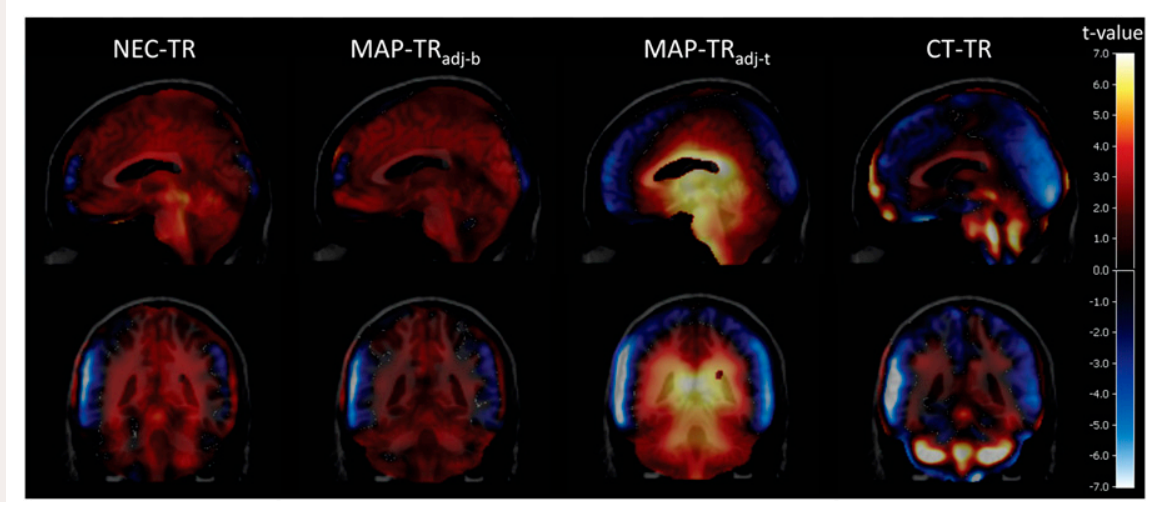




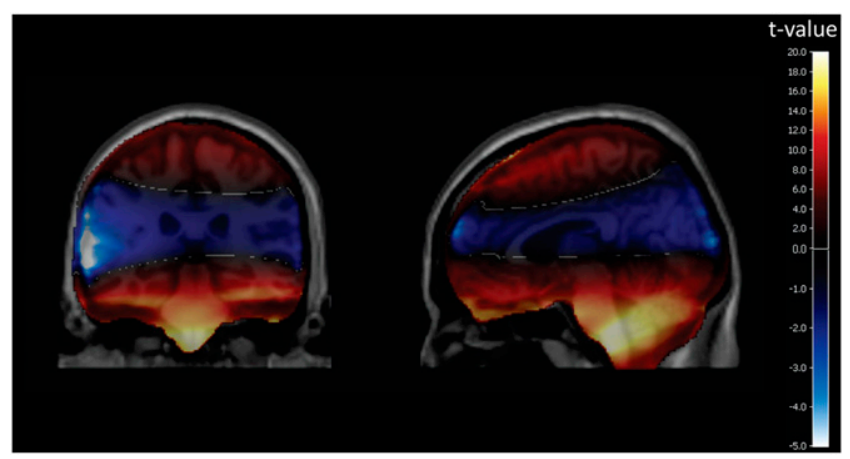

FIGURE 5. Effect of bone difference between CT-TR and MAP-TR def $\mu$-maps on reconstructed PET image. ACFs were calculated from segmented bones after removal of brain tissue parts of each $\mu$-map, and difference of ACFs was backprojected. Bone of CT-TR relatively increased signals of brain stem and cerebellum and decreased signals in central regions.

the CT-TR algorithm, as compared with the MAP-TR adj-t $_{\text {t }}$ algorithm, could more accurately correct the attenuation of the image. The CT-TR algorithm can represent, in the $\mu$-map, details of bones that not all other radionuclidebased transmission data could describe. These bones are characterized by thinness and low density. The complemented $\mu$-map for these bones showed increased intensity in the brain stem and cerebellum, as shown in Figure 5. More interestingly, intensity in the middle areas was decreased with the CT-TR algorithm, as compared with the MAP-TR $_{\text {def }}$ algorithm. This result may have been caused by a smeared and inaccurate $\mu$-valued skull with the MAP-TR $_{\text {def }}$ algorithm in the frontal and occipital regions. The result of Figure 5 is based only on a bone effect. The $\mu$-values for the tissue compartments are the same between the CT-TR and the MAP-TR def $_{\text {algorithms, }}$ as shown in Figure 5.

To summarize the results, the 2 effects are mingled for the CT-TR algorithm. One result of using the CT-TR algorithm is that the tissue effect makes the $\mu$-value of tissue more accurate than when using the MAP-TR $\mathrm{def}_{\mathrm{f}}$ algorithm. The other result of using the CT-TR algorithm is that the bone effect is a more accurate description of bone structures on the $\mu$-map than when using the MAP$\mathrm{TR}_{\mathrm{def}}$ algorithm. The tissue effect causes an increase in the intensity of the central and lower regions of the brain, and the bone effect causes a decrease in the intensity of the central regions and increases the intensity of the lower regions of the brain. Therefore, an intensity decrease due to the bone effect may suppress the intensity increase in the central region by the tissue effect, and the CT-TR algorithm - as compared with PET/CT-resulted in a betterbalanced intensity in the brain, as shown in Figure 3. These effects are verified by the comparison of Figures $3 \mathrm{~A}$ and 4D. The CT-TR algorithm almost exactly restores or suppresses the decreased or increased regions of the

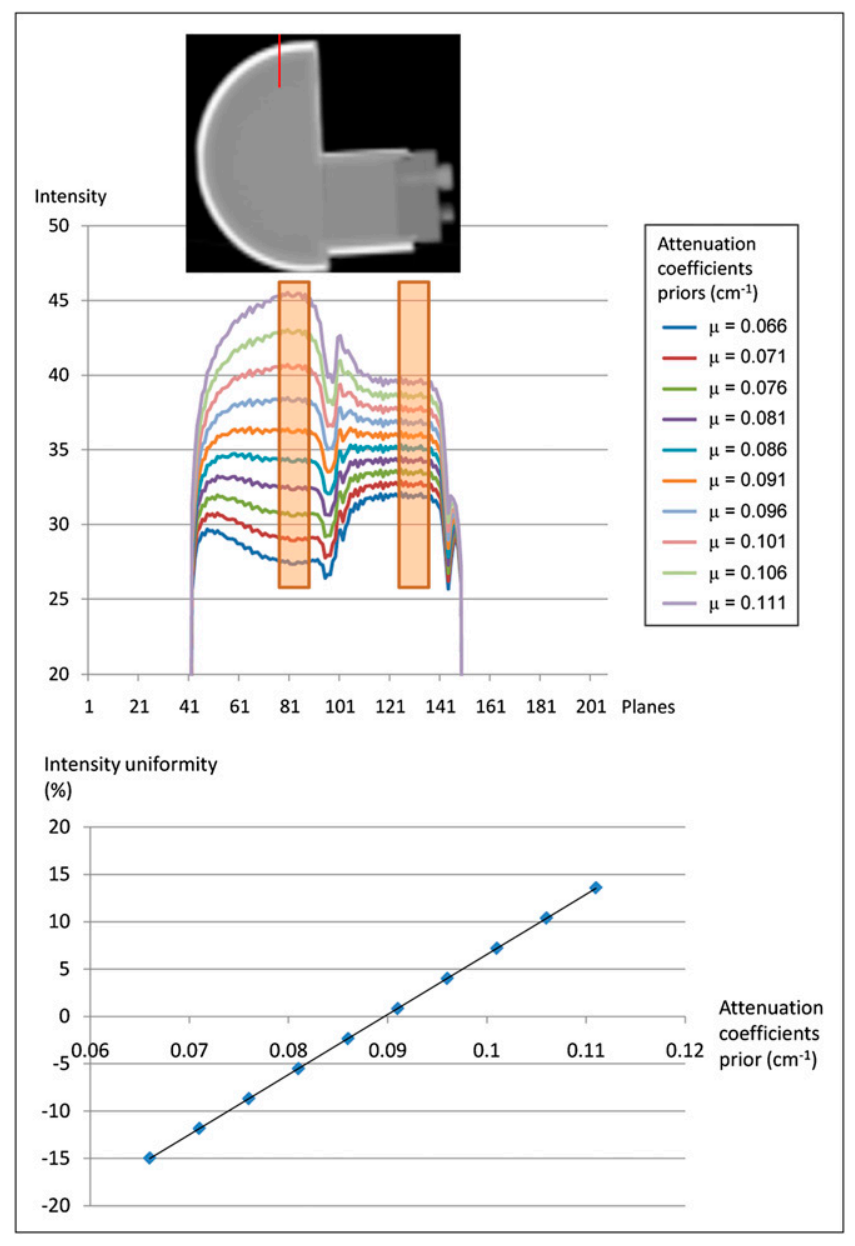

FIGURE 6. Effect of attenuation coefficient priors in MAPTR algorithm on reconstructed PET image. Overestimated $\mu$-priors increased intensity of PET image and vice versa. Increased or decreased change became larger when volume was larger.

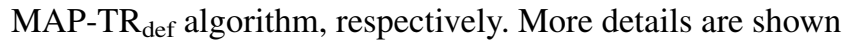
in Supplemental Figure 2.

Although the misestimation of a $\mu$-map is presumed to be the major cause of the negative bias for the cerebellum and brain stem, it is still unknown why other commercial PET systems previously developed do not have this bias. Therefore, differences between the HRRT PET system and other commercial systems should be noted. The HRRT system uses a ${ }^{137} \mathrm{Cs}$ point source and fanbeam scanning for the transmission scan; most of the other systems use a rotating ${ }^{68} \mathrm{Ge}$ rod source. The $\mu$-map of the ${ }^{137} \mathrm{Cs}$ point source is, however, known to have a signal-to-noise ratio superior to other radionuclide sources (17). Accordingly, for $\mu$-map quality, the transmission data of the HRRT is not the cause of the problem. Another difference between the HRRT and other commercial systems is the geometry of the gantry, with a lengthened bore of the HRRT in the axial direction. The axial FOV of most commercial PET scanners 
is approximately $16 \mathrm{~cm}$, but the axial FOV of the HRRT is approximately $25 \mathrm{~cm}$. Consequently, most $\mu$-maps for the commercial PET systems do not contain bone structures below the skull and only the skull is dominant on a $\mu$-map. The skull, as compared with the other bones below the skull, is not relatively influenced by the quality of the $\mu$-map because it has a uniform thickness and a simple shape. Although a radioisotope-based method, as compared with a CT-based method, cannot provide a precise $\mu$-map, the quality is sufficient for the skull region. The axial FOV of the HRRT includes many other bones with complicated structures, such as the teeth, jaw, and neck. However, these bones were out of the FOV for the $\mu$-map of existing PET systems. A lengthened axial FOV, consequently including the bone structures below the skull, might cause underestimation of the ACFs and inaccurate results.

Not only attenuation correction but also scatter correction will be influenced by transmission reconstruction for currently used PET systems, including the HRRT, because they estimate the scatter-correction factors using singlescatter simulation methods based on the $\mu$-map. We evaluated scatter correction, as compared with attenuation correction, by changing the $\mu$-map of the various algorithms, but the effect was not significant.

\section{CONCLUSION}

Among the proposed algorithms for transmission reconstruction, the CT-TR algorithm provided the most accurate attenuation correction for the HRRT system. The CT-TR algorithm, however, has some pitfalls, because the algorithm requires the installation of additional hardware for CT or scanning from another CT scanner. For the latter case, inaccurate coregistration may cause additional problems, and for the former case, additional costs will be incurred. In addition, metallic artifacts with $\mathrm{CT}$ are major weaknesses. The MAP-TR algorithm, with appropriate priors minimizing the bias, is another choice without any additional hardware installation for the system. A validation study of the optimized priors for each tissue component is necessary. A $\mu$-value of brain tissue of $0.0994 \mathrm{~cm}^{-1}$ is sufficient. However, more studies are needed to determine the $\mu$-value for bone. Finally, for a PET scanner with a long axial FOV, a new method should be proposed to describe thin or low-density bones for the transmission data, unless the PET scanner is equipped with CT.

\section{ACKNOWLEDGMENT}

This work was supported by the Basic Science Research Program through the National Research Foundation (NRF) funded by the Ministry of Education, Science and Technology (2008-04159 and 2009-0081933).

\section{REFERENCES}

1. Cho ZH, Chan JK, Eriksson L. Circular ring transverse axial positron camera for 3-dimensional reconstruction of radionuclide distribution. IEEE Trans Nucl Sci. 1976;23:613-623.

2. Phelps ME, Hoffman EJ, Mullani NA, Ter-Pogossian MM. Application of annihilation coincidence detection to transaxial reconstruction tomography. J Nucl Med. 1975;16:210-224.

3. Cho $\mathrm{ZH}$, Farukhi MR. Bismuth germanate as a potential scintillation detector in positron cameras. J Nucl Med. 1977;18:840-844.

4. Townsend DW, Geissbuhler A, Defrise M, et al. Fully three-dimensional reconstruction for a PET camera with retractable septa. IEEE Trans Med Imaging. 1991;10:505-512.

5. Wienhard K, Schmand M, Casey ME, et al. The ECAT HRRT: performance and first clinical application of the new high resolution research tomograph. IEEE Trans Nucl Sci. 2002;49:104-110.

6. Cherry SR, Meikle SR, Hoffman EJ. Correction and characterization of scattered events in three-dimensional PET using scanners with retractable septa. $J$ Nucl Med. 1993;34:671-678.

7. Nuyts J, Dupont P, Stroobants S, Maes A, Mortelmans L, Suetens P. Evaluation of maximum-likelihood based attenuation correction in positron emission tomography. IEEE Trans Nucl Sci. 1999;46:1136-1141.

8. van Velden FHP, Kloet RW, van Berckel BNM, et al. Impact of attenuation correction strategies on the quantification of High Resolution Research Tomograph PET studies. Phys Med Biol. 2008;53:99-118.

9. Nuyts J, Michel C, Dupont P. Maximum-likelihood expectation-maximization reconstruction of sinograms with arbitrary noise distribution using NECtransformations. IEEE Trans Med Imaging. 2001;20:365-375.

10. Litton JE, Nordstrom AL, Halldin C, Farde L. Attenuation correction in PETtransmission vs. contour finding in the study of $\left[{ }^{11} \mathrm{C}\right]$ raclopride binding. IEEE Conf Rec. 1993;3:1713-1716.

11. Zaidi H, Montandon ML, Slosman DO. Magnetic resonance imaging-guided attenuation and scatter corrections in three-dimensional brain positron emission tomography. Med Phys. 2003;30:937-948.

12. International Commission on Radiation Units and Measurements (ICRU). Tissue Substitute in Radiation Dosimetry and Measurement. Report 44. Bethesda, MD: ICRU; 1989.

13. Burger C, Goerres G, Schoenes S, Buck A, Lonn A, Von Schulthess G. PET attenuation coefficients from CT images: experimental evaluation of the transformation of CT into PET 511-keV attenuation coefficients. Eur J Nucl Med Mol Imaging. 2002;29:922-927.

14. Carney JPJ, Townsend DW, Rappoport V, Bendriem B. Method for transforming CT images for attenuation correction in PET/CT imaging. Med Phys. 2006;33: 976-983.

15. Hong IK, Chung ST, Kim HK, Kim YB, Son YD, Cho ZH. Ultra fast symmetry and SIMD-based projection-backprojection (SSP) algorithm for 3-D PET image reconstruction. IEEE Trans Med Imaging. 2007;26:789-803.

16. Watson CC, Newport D, Casey ME. A single scatter simulation technique for scatter correction in 3D PET. In: Grangeat P, Amans JL, eds. Three-Dimensional Image Reconstruction in Radiology and Nuclear Medicine. Dordrecht, The Netherlands: Kluwer Academic Publishers; 1996:255-268.

17. Knoess C, Rist J, Michel C, et al. Evaluation of single photon transmission for the HRRT. IEEE Nucl Sci Symp Conf Rec. 2003;3:1936-1940. 